\title{
Continued Fractions of Order Six and New Eisenstein Series Identities
}

\author{
Chandrashekar Adiga, A. Vanitha, and M. S. Surekha \\ Department of Studies in Mathematics, University of Mysore, Manasagangotri, Mysore 570 006, India \\ Correspondence should be addressed to Chandrashekar Adiga; cadiga@maths.uni-mysore.ac.in \\ Received 25 February 2014; Revised 25 April 2014; Accepted 7 May 2014; Published 27 May 2014 \\ Academic Editor: Ahmed Laghribi
}

Copyright (C) 2014 Chandrashekar Adiga et al. This is an open access article distributed under the Creative Commons Attribution License, which permits unrestricted use, distribution, and reproduction in any medium, provided the original work is properly cited.

We prove two identities for Ramanujan's cubic continued fraction and a continued fraction of Ramanujan, which are analogues of Ramanujan's identities for the Rogers-Ramanujan continued fraction. We further derive Eisenstein series identities associated with Ramanujan's cubic continued fraction and Ramanujan's continued fraction of order six.

\section{Introduction}

Throughout this paper, we assume that $|q|<1$ and for each positive integer $n$, we use the standard product notation

$$
\begin{aligned}
& (a ; q)_{0}:=1, \quad(a ; q)_{n}:=\prod_{j=0}^{n-1}\left(1-a q^{j}\right), \quad n \geq 1, \\
& (a ; q)_{\infty}:=\prod_{j=0}^{\infty}\left(1-a q^{j}\right) .
\end{aligned}
$$

Srinivasa Ramanujan made some significant contributions to the theory of continued fraction expansions. The most beautiful continued fraction expansions can be found in Chapters 12 and 16 of his second notebook [1].

The celebrated Rogers-Ramanujan continued fraction is defined by [2]

$$
R(q):=q^{1 / 5} \frac{f\left(-q,-q^{4}\right)}{f\left(-q^{2},-q^{3}\right)}=\frac{q^{1 / 5}}{1+\frac{q}{1+\frac{q^{2}}{1+\frac{q^{3}}{1+\cdots}}}},
$$

where

$$
\begin{aligned}
f(a, b)= & \sum_{n=-\infty}^{\infty} a^{n(n+1) / 2} b^{n(n-1) / 2}, \\
= & 1+\sum_{n=1}^{\infty}(a b)^{n(n-1) / 2}\left(a^{n}+b^{n}\right), \\
& |a b|<1,
\end{aligned}
$$

is Ramanujan's general theta function.

Ramanujan eventually found several generalizations and ramifications of $R(q)$ which can be found in his notebooks [1] and "lost notebook" [3]. Recently, Liu [4] and Chan et al. [5] have established several new identities associated with the Rogers-Ramanujan continued fraction $R(q)$ including Eisenstein series identities involving $R(q)$.

The beautiful Ramanujan's cubic continued fraction $G(q)$, first introduced by Srinivasa Ramanujan in his second letter to Hardy [2, page xxvii], is defined by

$$
\begin{aligned}
G(q):=q^{1 / 3} \frac{f\left(-q,-q^{5}\right)}{f\left(-q^{3},-q^{3}\right)} & \\
= & \frac{q^{1 / 3}}{1+\frac{q+q^{2}}{1+\frac{q^{2}+q^{4}}{1+\frac{q^{3}+q^{6}}{1+\cdots}}}} .
\end{aligned}
$$


Adiga et al. [6], Bhargava et al. [7], Chan [8], and Vasuki et al. [9] have proved several elegant theorems for $G(q)$, many of which are analogues of well-known properties satisfied by the Rogers-Ramanujan continued fraction.

Recently, Vasuki et al. [10] have studied the following continued fraction of order six:

$$
\begin{aligned}
X(q) & :=q^{1 / 4} \frac{f\left(-q,-q^{5}\right)}{f\left(-q^{2},-q^{4}\right)} \\
= & \frac{q^{1 / 4}\left(1-q^{-2}\right)}{\left(1-q^{-3 / 2}\right)+\frac{\left(q^{1 / 4}-q^{-1 / 4}\right)\left(q^{-7 / 4}-q^{7 / 4}\right)}{\left(1-q^{-3 / 2}\right)\left(1+q^{3}\right)+\cdots}} .
\end{aligned}
$$

The continued fraction (5) is a special case of a fascinating continued fraction identity recorded by Ramanujan in his second notebook [1], [11, page 24]. Furthermore, they have established modular relations between the continued fractions $X(q)$ and $X\left(q^{n}\right)$ for $n=2,3,5,7$, and 11 .

In Section 3 of this paper, we establish two new identities associated with the continued fractions $G(q)$ and $X(q)$, using the quintuple product identity. In Section 4 , we derive Eisenstein series identities associated with $G(q)$ and $X(q)$.

\section{Definitions and Preliminary Results}

In this section, we present some basic definitions and preliminary results. One of the most interesting special cases of $f(a, b)$ is [11, Entry 22]

$$
f(-q):=f\left(-q,-q^{2}\right)=\sum_{n=-\infty}^{\infty}(-1)^{n} q^{n(3 n-1) / 2}=(q ; q)_{\infty} .
$$

Note that the Dedekind eta function $\eta(\tau)=q^{1 / 24} f(-q)$, where $q=e^{2 \pi i \tau}$, $\operatorname{Im} \tau>0$. We need the following three lemmas to prove our main results.

Lemma 1 (see [11, Entry 30, page 46]). One has

$$
f(a, b)+f(-a,-b)=2 f\left(a^{3} b, a b^{3}\right) .
$$

Lemma 2 (see [11, page 80$]$ ). One has

$$
\begin{gathered}
f\left(B^{3} q, \frac{q^{5}}{B^{3}}\right)-B^{2} f\left(\frac{q}{B^{3}}, B^{3} q^{5}\right) \\
=f\left(-q^{2}\right) \frac{f\left(-B^{2},-q^{2} / B^{2}\right)}{f(B q, q / B)} .
\end{gathered}
$$

Lemma 3 (see [12, Lemma 2(ii)]). Let $m=[s /(s-r)], l=$ $m(s-r)-r, k=-m(s-r)+s$, and $h=m r-(m(m-1)(s-r)) / 2$, $0 \leq r<s$. Here $[x]$ denotes the largest integer less than or equal to $x$. Then

(i) $f\left(q^{-r}, q^{s}\right)=q^{-h} f\left(q^{l}, q^{k}\right)$;

(ii) $f\left(-q^{-r},-q^{s}\right)=(-1)^{m} q^{-h} f\left(-q^{l},-q^{k}\right)$.

\section{Main Results}

The Jacobi triple product identity states that

$$
\sum_{n=-\infty}^{\infty}(-1)^{n} q^{n(n-1) / 2} z^{n}=(q ; q)_{\infty}(z ; q)_{\infty}\left(\frac{q}{z} ; q\right)_{\infty}, \quad z \neq 0 .
$$

In Ramanujan's notation, the Jacobi triple product identity takes the form

$$
f(a, b)=(-a ; a b)_{\infty}(-b ; a b)_{\infty}(a b ; a b)_{\infty} .
$$

The Jacobi triple product identity was first proved by Gauss [13]. Using (3), we have

$$
f\left(-e^{2 i z},-q e^{-2 i z}\right)=2 i e^{i z} \sum_{n=0}^{\infty}(-1)^{n+1} q^{n(n+1) / 2} \sin (2 n+1) z .
$$

Putting $a=-e^{2 i z}$ and $b=-q e^{-2 i z}$ in (10), we obtain

$f\left(-e^{2 i z},-q e^{-2 i z}\right)=\left(1-e^{2 i z}\right)\left(q e^{2 i z} ; q\right)_{\infty}\left(q e^{-2 i z} ; q\right)_{\infty}(q ; q)_{\infty}$.

Putting $z=\pi / 6$ and $z=2 \pi / 6$, respectively, in (12), we obtain

$$
\begin{aligned}
& f\left(-e^{i \pi / 3},-q e^{-i \pi / 3}\right) \\
& =\left(1-e^{i \pi / 3}\right)\left(q e^{i \pi / 3} ; q\right)_{\infty}\left(q e^{-i \pi / 3} ; q\right)_{\infty}(q ; q)_{\infty}, \\
& f\left(-e^{i 2 \pi / 3},-q e^{-i 2 \pi / 3}\right) \\
& =\left(1-e^{i 2 \pi / 3}\right)\left(q e^{i 2 \pi / 3} ; q\right)_{\infty}\left(q e^{-i 2 \pi / 3} ; q\right)_{\infty}(q ; q)_{\infty} .
\end{aligned}
$$

Multiplying (13) and (14) together and using the identities

$$
\begin{gathered}
\left(1-e^{i \pi / 3}\right)\left(1-e^{i 2 \pi / 3}\right)=-i \sqrt{3}, \\
(1-x)\left(1-x e^{2 \pi i / 6}\right)\left(1-x e^{-2 \pi i / 6}\right)\left(1-x e^{4 \pi i / 6}\right) \\
\times\left(1-x e^{-4 \pi i / 6}\right)\left(1-x e^{6 \pi i / 6}\right)=\left(1-x^{6}\right),
\end{gathered}
$$

in the resulting equation and then after some simplifications, we obtain the following identity:

$$
\begin{gathered}
f\left(-e^{i \pi / 3},-q e^{-i \pi / 3}\right) f\left(-e^{i 2 \pi / 3},-q e^{-i 2 \pi / 3}\right) \\
=-i \sqrt{3} q^{-1 / 4} \frac{\eta^{2}(\tau) \eta(6 \tau)}{\eta(2 \tau)} .
\end{gathered}
$$

Theorem 4. Let $|q|<1, \alpha=-1$, and $\beta=1$. Then

$$
\begin{aligned}
& \prod_{n=1}^{\infty} \frac{1}{\left(1+\alpha q^{n / 2}+q^{n}\right)}-\prod_{n=1}^{\infty} \frac{1}{\left(1+\beta q^{n / 2}+q^{n}\right)} \\
& \quad=4 q^{1 / 24} \frac{\eta(\tau) \eta^{2}(3 \tau)}{\eta(\tau) \eta^{2}(3 \tau / 2)+\eta^{2}(\tau / 2) \eta(3 \tau)} G(q), \\
& \prod_{n=1}^{\infty} \frac{1}{\left(1+\alpha q^{n / 2}+q^{n}\right)}-\prod_{n=1}^{\infty} \frac{1}{\left(1+\beta q^{n / 2}+q^{n}\right)} \\
& =4 q^{1 / 24} \frac{\eta(\tau) \eta(2 \tau) \eta(6 \tau)}{\eta(\tau) \eta^{2}(3 \tau / 2)+\eta^{2}(\tau / 2) \eta(3 \tau)} X(q) .
\end{aligned}
$$


Proof. We may rewrite (13) and (14) as follows:

$$
\begin{aligned}
& f\left(-e^{i \pi / 3},-q e^{-i \pi / 3}\right) \\
& =\left(1-e^{i \pi / 3}\right) q^{-1 / 24} \eta(\tau) \prod_{n=1}^{\infty}\left(1+\alpha q^{n}+q^{2 n}\right), \\
& f\left(-e^{i 2 \pi / 3},-q e^{-i 2 \pi / 3}\right) \\
& =\left(1-e^{i 2 \pi / 3}\right) q^{-1 / 24} \eta(\tau) \prod_{n=1}^{\infty}\left(1+\beta q^{n}+q^{2 n}\right),
\end{aligned}
$$

where

$$
\alpha=-2 \cos \frac{\pi}{3}=-1, \quad \beta=-2 \cos \frac{2 \pi}{3}=1 .
$$

Then,

$$
\begin{aligned}
& \prod_{n=1}^{\infty}\left(1+\alpha q^{n}+q^{2 n}\right)=\frac{q^{1 / 24}}{\eta(\tau)} \frac{f\left(-e^{i \pi / 3},-q e^{-i \pi / 3}\right)}{\left(1-e^{i \pi / 3}\right)}, \\
& \prod_{n=1}^{\infty}\left(1+\beta q^{n}+q^{2 n}\right)=\frac{q^{1 / 24}}{\eta(\tau)} \frac{f\left(-e^{i 2 \pi / 3},-q e^{-i 2 \pi / 3}\right)}{\left(1-e^{i 2 \pi / 3}\right)} .
\end{aligned}
$$

Multiplying the above two equations together and then using (16), in resulting identity, we find that

$$
\prod_{n=1}^{\infty}\left(1+\alpha q^{n}+q^{2 n}\right)\left(1+\beta q^{n}+q^{2 n}\right)=q^{-1 / 6} \frac{\eta(6 \tau)}{\eta(2 \tau)} .
$$

Subtracting (22) from (21), we obtain

$$
\begin{aligned}
& \prod_{n=1}^{\infty}\left(1+\alpha q^{n}+q^{2 n}\right)-\prod_{n=1}^{\infty}\left(1+\beta q^{n}+q^{2 n}\right) \\
& \quad=\frac{q^{1 / 24}}{\eta(\tau)}\left(\frac{f\left(-e^{i \pi / 3},-q e^{-i \pi / 3}\right)}{\left(1-e^{i \pi / 3}\right)}-\frac{f\left(-e^{i 2 \pi / 3},-q e^{-i 2 \pi / 3}\right)}{\left(1-e^{i 2 \pi / 3}\right)}\right) .
\end{aligned}
$$

Using (11) in (24), we deduce that

$$
\begin{gathered}
\prod_{n=1}^{\infty}\left(1+\alpha q^{n}+q^{2 n}\right)-\prod_{n=1}^{\infty}\left(1+\beta q^{n}+q^{2 n}\right) \\
=\frac{q^{-1 / 12}}{\eta(\tau)} \sum_{n=0}^{\infty}(-1)^{n} P(n) q^{(2 n+1)^{2} / 8},
\end{gathered}
$$

where

$$
P(n)=\frac{2 \sin (2 n+1)(\pi / 6)}{i e^{-i \pi / 6}\left(1-e^{i \pi / 3}\right)}-\frac{2 \sin (2 n+1)(2 \pi / 6)}{i e^{-i 2 \pi / 6}\left(1-e^{i 2 \pi / 3}\right)} .
$$

Now, by direct computations, we find that

$$
\begin{array}{lr}
P(6 m+0)=0, & P(6 m+1)=2, \\
P(6 m+2)=2, & P(6 m+3)=-2, \\
P(6 m+4)=-2, & P(6 m+5)=0 .
\end{array}
$$

Therefore,

$$
\begin{aligned}
& \sum_{n=0}^{\infty}(-1)^{n} P(n) q^{(2 n+1)^{2} / 8} \\
&=2\left\{-\sum_{m=0}^{\infty} q^{(12 m+3)^{2} / 8}+\sum_{m=0}^{\infty} q^{(12 m+5)^{2} / 8}\right. \\
&\left.+\sum_{m=0}^{\infty} q^{(12 m+7)^{2} / 8}-\sum_{m=0}^{\infty} q^{(12 m+9)^{2} / 8}\right\} .
\end{aligned}
$$

In the right-hand side of the above equation, changing $m$ to $m-1$ in the first two summations and also changing $m$ to $-m$ in the last two summations, we obtain

$$
\begin{aligned}
& \sum_{n=0}^{\infty}(-1)^{n} P(n) q^{(2 n+1)^{2} / 8} \\
& \quad=2\left\{\sum_{m=-\infty}^{\infty} q^{(12 m-7)^{2} / 8}-\sum_{m=-\infty}^{\infty} q^{(12 m-9)^{2} / 8}\right\} .
\end{aligned}
$$

Now, using the definition of $f(a, b)$ in the right-hand side of the above equation, we find that

$$
\begin{aligned}
& \sum_{n=0}^{\infty}(-1)^{n} P(n) q^{(2 n+1)^{2} / 8} \\
& \quad=-2 q^{9 / 8}\left\{f\left(q^{9}, q^{27}\right)-q^{2} f\left(q^{3}, q^{33}\right)\right\} .
\end{aligned}
$$

In the quintuple product identity (8), replacing $q$ by $q^{6}$ and then setting $B=q$, we find that

$$
f\left(q^{9}, q^{27}\right)-q^{2} f\left(q^{3}, q^{33}\right)=\frac{f\left(-q^{12}\right) f\left(-q^{2},-q^{10}\right)}{f\left(q^{5}, q^{7}\right)} \text {. }
$$

Combining (25), (30), and (31), we find that

$$
\begin{aligned}
\prod_{n=1}^{\infty}(1 & \left.+\alpha q^{n}+q^{2 n}\right)-\prod_{n=1}^{\infty}\left(1+\beta q^{n}+q^{2 n}\right) \\
& =\frac{-2 q^{25 / 24}}{\eta(\tau)}\left\{\frac{f\left(-q^{12}\right) f\left(-q^{2},-q^{10}\right)}{f\left(q^{5}, q^{7}\right)}\right\} .
\end{aligned}
$$

Dividing both sides of (32) by $\prod_{n=1}^{\infty}\left(1+\alpha q^{n}+q^{2 n}\right)\left(1+\beta q^{n}+q^{2 n}\right)$ and then using (23), we obtain

$$
\begin{aligned}
\prod_{n=1}^{\infty}(1 & \left.+\alpha q^{n}+q^{2 n}\right)^{-1}-\prod_{n=1}^{\infty}\left(1+\beta q^{n}+q^{2 n}\right)^{-1} \\
& =2 q^{29 / 24} \frac{\eta(2 \tau)}{\eta(\tau) \eta(6 \tau)}\left\{\frac{f\left(-q^{12}\right) f\left(-q^{2},-q^{10}\right)}{f\left(q^{5}, q^{7}\right)}\right\} .
\end{aligned}
$$

Replacing $a$ by $q$ and $b$ by $q^{2}$ in (7), we find that

$$
f\left(q, q^{2}\right)+f\left(-q,-q^{2}\right)=2 f\left(q^{5}, q^{7}\right) .
$$


Now, using the above equation in the right-hand side of (33) and then changing $q$ to $q^{1 / 2}$ throughout, we obtain (17). Equation (18) follows from the following identity:

$$
G(q)=\frac{\eta(2 \tau) \eta(6 \tau)}{\eta^{2}(3 \tau)} X(q)
$$

This completes the proof of Theorem 4.

\section{Eisenstein Series Identities Associated with $G(q)$ and $X(q)$}

In this section, we present four Eisenstein series identities associated with $G(q)$ and $X(q)$.

Theorem 5. Let $|q|<1$. Then

$$
\begin{aligned}
& \sum_{\substack{n=1 \\
n \equiv 1(\bmod 3)}}^{\infty} \frac{q^{n}-q^{3 n}+q^{5 n}}{1-q^{6 n}}-\sum_{\substack{n=1 \\
n \equiv 2(\bmod 3)}}^{\infty} \frac{q^{n}-q^{3 n}+q^{5 n}}{1-q^{6 n}} \\
& \quad=\frac{\eta^{3}(18 \tau)}{\eta(6 \tau)}\left[\frac{1}{G\left(q^{3}\right)}-1-G\left(q^{3}\right)\right] .
\end{aligned}
$$

Proof. Changing $n$ to $-n$ in the second summation, of the lefthand side of Theorem 5, we have

$$
\begin{aligned}
& \sum_{\substack{n=1 \\
n \equiv 1(\bmod 3)}}^{\infty} \frac{q^{n}-q^{3 n}+q^{5 n}}{1-q^{6 n}}-\sum_{\substack{n=1 \\
n \equiv 2(\bmod 3)}}^{\infty} \frac{q^{n}-q^{3 n}+q^{5 n}}{1-q^{6 n}} \\
& =\sum_{\substack{n=1 \\
n \equiv 1(\bmod 3)}}^{\infty} \frac{q^{n}-q^{3 n}+q^{5 n}}{1-q^{6 n}} \\
& \quad-\sum_{\substack{n=-\infty \\
n \equiv 1(\bmod 3)}}^{-1} \frac{q^{-n}-q^{-3 n}+q^{-5 n}}{1-q^{-6 n}} \\
& =\sum_{n=-\infty}^{\infty} \frac{q^{3 n+1}}{1-q^{18 n+6}}-\sum_{n=-\infty}^{\infty} \frac{q^{9 n+3}}{1-q^{18 n+6}}+\sum_{n=-\infty}^{\infty} \frac{q^{15 n+5}}{1-q^{18 n+6}} .
\end{aligned}
$$

Using a corollary of Ramanujan's ${ }_{1} \Psi_{1}$ summation formula [11, Entry 17, page 32]

$$
\sum_{n=-\infty}^{\infty} \frac{z^{n}}{1-a q^{n}}=\frac{(a z, q / a z, q, q ; q)_{\infty}}{(a, q / a, z, q / z ; q)_{\infty}}, \quad|q|<|z|<1,
$$

and Lemma 3 in (37), we find that

$$
\begin{gathered}
\sum_{\substack{n=1 \\
n \equiv 1(\bmod 3)}}^{\infty} \frac{q^{n}-q^{3 n}+q^{5 n}}{1-q^{6 n}}-\sum_{\substack{n=1 \\
n=2(\bmod 3)}}^{\infty} \frac{q^{n}-q^{3 n}+q^{5 n}}{1-q^{6 n}} \\
=q \frac{\left(q^{9}, q^{9}, q^{18}, q^{18} ; q^{18}\right)_{\infty}}{\left(q^{6}, q^{12}, q^{3}, q^{15} ; q^{18}\right)_{\infty}}-q^{3} \frac{\left(q^{15}, q^{3}, q^{18}, q^{18} ; q^{18}\right)_{\infty}}{\left(q^{6}, q^{12}, q^{9}, q^{9} ; q^{18}\right)_{\infty}} \\
\quad+q^{5} \frac{\left(q^{21}, q^{-3}, q^{18}, q^{18} ; q^{18}\right)_{\infty}}{\left(q^{6}, q^{12}, q^{15}, q^{3} ; q^{18}\right)_{\infty}} \\
=\frac{\left(q^{18} ; q^{18}\right)_{\infty}^{2}}{\left(q^{6}, q^{12} ; q^{18}\right)_{\infty}}\left\{q \frac{f\left(-q^{9},-q^{9}\right)}{f\left(-q^{3},-q^{15}\right)}-q^{3} \frac{f\left(-q^{3},-q^{15}\right)}{f\left(-q^{9},-q^{9}\right)}\right. \\
\left.-q^{2} \frac{f\left(-q^{3},-q^{15}\right)}{f\left(-q^{3},-q^{15}\right)}\right\} .
\end{gathered}
$$

Using (4) in (39) and after some simplifications, we obtain (36).

Theorem 6. Let $|q|<1$. Then

$$
\begin{aligned}
& \sum_{\substack{n=1 \\
n=1(\bmod 6)}}^{\infty} \frac{q^{n}-q^{2 n}-q^{4 n}+q^{5 n}}{1-q^{6 n}} \\
& -\sum_{\substack{n=1 \\
n=5(\bmod 6)}}^{\infty} \frac{q^{n}-q^{2 n}-q^{4 n}+q^{5 n}}{1-q^{6 n}} \\
& =\frac{\eta(12 \tau) \eta(18 \tau) \eta(36 \tau)}{\eta(6 \tau)} \\
& \quad \times\left[\frac{1}{X\left(q^{6}\right)}-\frac{\eta^{2}(18 \tau)}{\eta(12 \tau) \eta(36 \tau)}-X\left(q^{6}\right)\right] .
\end{aligned}
$$

Proof. Using the identity

$$
\begin{aligned}
& \sum_{n=0}^{\infty} \frac{x^{n}}{1-y q^{n}} \\
& \quad=\sum_{n=0}^{\infty} \sum_{m=0}^{\infty} x^{n} y^{m} q^{m n}=\sum_{n=0}^{\infty} \frac{y^{n}}{1-x q^{n}}, \quad|x|,|y|<1,
\end{aligned}
$$

the left-hand side of Theorem 6 can be written as

$$
\begin{aligned}
& \sum_{\substack{n=1 \\
n \equiv 1(\bmod 6)}}^{\infty} \frac{q^{n}-q^{2 n}-q^{4 n}}{1-q^{6 n}}-\sum_{\substack{n=-\infty \\
n=1(\bmod 6)}}^{-1} \frac{-q^{-2 n}-q^{-4 n}+q^{-5 n}}{1-q^{-6 n}} \\
& =\sum_{n=-\infty}^{\infty} \frac{q^{6 n+1}}{1-q^{36 n+6}}-\sum_{n=-\infty}^{\infty} \frac{q^{12 n+2}}{1-q^{36 n+6}}-\sum_{n=-\infty}^{\infty} \frac{q^{24 n+4}}{1-q^{36 n+6}} .
\end{aligned}
$$


Using (38) in (42), we obtain

$$
\begin{aligned}
& \sum_{\substack{n=1 \\
n \equiv 1(\bmod 6)}}^{\infty} \frac{q^{n}-q^{2 n}-q^{4 n}+q^{5 n}}{1-q^{6 n}} \\
& -\sum_{\substack{n=1 \\
n \equiv 5(\bmod 6)}}^{\infty} \frac{q^{n}-q^{2 n}-q^{4 n}+q^{5 n}}{1-q^{6 n}} \\
& =\frac{\left(q^{36} ; q^{36}\right)_{\infty}^{2}}{\left(q^{6}, q^{30} ; q^{36}\right)_{\infty}} \\
& \left\{q^{\left(q^{12}, q^{24} ; q^{36}\right)_{\infty}} \frac{\left(q^{6}, q^{30} ; q^{36}\right)_{\infty}}{q^{2}} \frac{\left(q^{18}, q^{18} ; q^{36}\right)_{\infty}}{\left(q^{12}, q^{24} ; q^{36}\right)_{\infty}}\right. \\
& \left.\quad-q^{4} \frac{\left(q^{6}, q^{30} ; q^{36}\right)_{\infty}}{\left(q^{12}, q^{24} ; q^{36}\right)_{\infty}}\right\} .
\end{aligned}
$$

Using (5) in (43) and after some simplifications, we obtain (40). yield

Differentiating both sides of (12) and then setting $z=0$

$$
f^{\prime}(-1,-q)=-2 i(q ; q)_{\infty}^{3}
$$

where $f^{\prime}$ denotes the partial derivative of $f$ with respect to $z$.

Now we prove a lemma, which is useful to prove Eisenstein series identities associated with $G(q)$ and $X(q)$.

Lemma 7. Consider the following:

$$
\begin{aligned}
\sum_{n=1}^{\infty} \frac{q^{n}-}{q^{2 n}-q^{4 n}+q^{5 n}} & \sin 2 n z \\
= & \left(-q^{6 n} e^{2 i z} f^{\prime}\left(-1,-q^{6}\right) f\left(-q^{3},-q^{3}\right) f\left(-q,-q^{5}\right)\right. \\
& \left.\times f\left(-e^{4 i z},-q^{6} e^{-4 i z}\right)\right) \\
& \times\left(4 q^{2} f\left(-q e^{2 i z},-q^{5} e^{-2 i z}\right)\right. \\
& \left.\times f\left(-q^{-1} e^{2 i z},-q^{7} e^{-2 i z}\right) f\left(-q^{2} e^{2 i z},-q^{4} e^{-2 i z}\right)\right)^{-1} \\
& \times \frac{1}{f\left(-q^{-2} e^{2 i z},-q^{8} e^{-2 i z}\right)} .
\end{aligned}
$$

Proof. For simplicity, we use $F(z, q)$ to denote the logarithmic derivative of $i q^{1 / 8} e^{-i z} f\left(-e^{2 i z},-q e^{-2 i z}\right)$ with respect to $z$. To prove this lemma we need the following identity, which can be found in [14, Theorem 5], [15, Corollary 2]:

$$
\begin{gathered}
F\left(z_{1}, q\right)+F\left(z_{2}, q\right)+F\left(z_{3}, q\right)-F\left(z_{1}+z_{2}+z_{3}, q\right) \\
=\left(-f^{\prime}(-1,-q) f\left(-e^{2 i\left(z_{1}+z_{2}\right)},-q e^{-2 i\left(z_{1}+z_{2}\right)}\right)\right. \\
\left.\quad \times f\left(-e^{2 i\left(z_{2}+z_{3}\right)},-q e^{-2 i\left(z_{2}+z_{3}\right)}\right)\right)
\end{gathered}
$$

$$
\begin{aligned}
& \times\left(f\left(-e^{2 i z_{1}},-q e^{-2 i z_{1}}\right)\right. \\
& \left.\times f\left(-e^{2 i z_{2}},-q e^{-2 i z_{2}}\right) f\left(-e^{2 i z_{3}},-q e^{-2 i z_{3}}\right)\right)^{-1} \\
& \times \frac{f\left(-e^{2 i\left(z_{1}+z_{3}\right)},-q e^{-2 i\left(z_{1}+z_{3}\right)}\right)}{f\left(-e^{2 i\left(z_{1}+z_{2}+z_{3}\right)},-q e^{-2 i\left(z_{1}+z_{2}+z_{3}\right)}\right)} .
\end{aligned}
$$

As the proof of this lemma is similar to that of Lemma 1 in [4], we omit the details.

Using (45), we derive the following Eisenstein series identity.

Theorem 8. Let $|q|<1$. Then

$$
\begin{aligned}
& \sum_{n=1}^{\infty} \frac{n\left(q^{n}-q^{2 n}-q^{4 n}+q^{5 n}\right)}{1-q^{6 n}} \\
& \quad=\frac{\eta^{6}(3 \tau)}{\eta^{2}(\tau)} G(q)=\frac{\eta^{4}(3 \tau) \eta(6 \tau) \eta(2 \tau)}{\eta^{2}(\tau)} X(q) .
\end{aligned}
$$

Proof. Dividing both sides of (45) by $z$ and then letting $z \rightarrow$ 0 , we obtain

$$
\begin{aligned}
\sum_{n=1}^{\infty} & \frac{n\left(q^{n}-q^{2 n}-q^{4 n}+q^{5 n}\right)}{1-q^{6 n}} \\
= & \left(\left(q^{6} ; q^{6}\right)_{\infty}^{6} f\left(-q^{3},-q^{3}\right) f\left(-q^{1},-q^{5}\right)\right) \\
& \times\left(q^{2} f\left(-q^{1},-q^{5}\right) f\left(-q^{-1},-q^{7}\right)\right. \\
& \left.\times f\left(-q^{2},-q^{4}\right) f\left(-q^{-2},-q^{8}\right)\right)^{-1} .
\end{aligned}
$$

Using Lemma 3 in (48), we complete the proof of Theorem 8.

We use $(\cdot / p)$ to denote the Legendre symbol modulo $p$. Setting $z=\pi / 3$ in (45) and noting that $\sin (2 n \pi / 3)=$ $(\sqrt{3} / 2)(n / 3)$, we find that

$$
\begin{aligned}
\frac{\sqrt{3}}{2} \sum_{n=1}^{\infty}\left(\frac{n}{3}\right) \frac{q^{n}-q^{2 n}-q^{4 n}+q^{5 n}}{1-q^{6 n}} & \left(-e^{2 i \pi / 3} f^{\prime}\left(-1,-q^{6}\right) f\left(-q^{3},-q^{3}\right)\right. \\
\quad & \left.\quad f\left(-q^{1},-q^{5}\right) f\left(-e^{4 i \pi / 3},-q^{6} e^{-4 i \pi / 3}\right)\right) \\
\times & \left(4 q^{2} f\left(-e^{2 i((\pi / 3)+\pi \tau)},-q^{6} e^{-2 i((\pi / 3)+\pi \tau)}\right)\right. \\
& \left.\times f\left(-e^{2 i((\pi / 3)-\pi \tau)},-q^{6} e^{-2 i((\pi / 3)-\pi \tau)}\right)\right)^{-1} \\
\times & (1)\left(f\left(-e^{2 i((\pi / 3)+2 \pi \tau)},-q^{6} e^{-2 i((\pi / 3)+2 \pi \tau)}\right)\right. \\
& \left.\times f\left(-e^{2 i((\pi / 3)-2 \pi \tau)},-q^{6} e^{-2 i((\pi / 3)-2 \pi \tau)}\right)\right)^{-1} .
\end{aligned}
$$


Recall the identity [16, Eq. (3. 1)]

$$
\begin{aligned}
f( & \left.-e^{2 i((\pi / 3)-z)},-q e^{-2 i((\pi / 3)-z)}\right) \\
& \times f\left(-e^{2 i((\pi / 3)+z)},-q e^{-2 i((\pi / 3)+z)}\right) \\
= & \frac{e^{-2 i z}(q ; q)_{\infty}^{3}}{e^{(-i 2 \pi) / 3}\left(q^{3} ; q^{3}\right)_{\infty}} \frac{f\left(-e^{6 i z},-q^{3} e^{-6 i z}\right)}{f\left(-e^{2 i z},-q e^{-2 i z}\right)} .
\end{aligned}
$$

Using the above identity in (49), we obtain the following Eisenstein series identity.

Theorem 9. Let $|q|<1$. Then

$$
\begin{aligned}
\sum_{n=1}^{\infty} & \left(\frac{n}{3}\right) \frac{q^{n}-q^{2 n}-q^{4 n}+q^{5 n}}{1-q^{6 n}} \\
& =\frac{\eta^{2}(3 \tau) \eta(\tau) \eta(9 \tau) \eta(18 \tau)}{\eta^{3}(6 \tau)} G(q) \\
& =\frac{\eta(\tau) \eta(2 \tau) \eta(9 \tau) \eta(18 \tau)}{\eta^{2}(6 \tau)} X(q) .
\end{aligned}
$$

\section{Conflict of Interests}

The authors declare that there is no conflict of interests regarding the publication of this paper.

\section{Acknowledgments}

The authors would like to thank the referees for their several helpful comments and suggestions. The first author is thankful to the University Grants Commission, Government of India, for the financial support under the Grant F.510/2/SAPDRS/2011. The second author is thankful to DST, New Delhi, for awarding INSPIRE Fellowship (no. DST/INSPIRE Fellowship/2012/122). The third author is thankful to UGC, New Delhi, for UGC-BSR fellowship, under which this work has been done.

\section{References}

[1] S. Ramanujan, Notebooks. Vols. 1, 2, Tata Institute of Fundamental Research, Bombay, India, 1957.

[2] S. Ramanujan, Collected Papers, Cambridge University Press, Cambridge, UK, 1927, repriented by Chelsea, New York, NY, USA, 1962; reprinted by the American Mathematical Society, Providence, RI, USA, 2000.

[3] S. Ramanujan, The Lost Notebook and Other Unpublished Papers, Springer, Berlin, Germany; Narosa Publishing House, New Delhi, India, 1988.

[4] Z.-G. Liu, "A theta function identity and the Eisenstein series on $\Gamma_{0}(5)$," Journal of the Ramanujan Mathematical Society, vol. 22, no. 3, pp. 283-298, 2007.

[5] H. H. Chan, S. H. Chan, and Z.-G. Liu, "The Rogers-Ramanujan continued fraction and a new Eisenstein series identity," Journal of Number Theory, vol. 129, no. 7, pp. 1786-1797, 2009.

[6] C. Adiga, T. Kim, M. S. Mahadeva Naika, and H. S. Madhusudhan, "On Ramanujan's cubic continued fraction and explicit evaluations of theta-functions," Indian Journal of Pure and Applied Mathematics, vol. 35, no. 9, pp. 1047-1062, 2004.

[7] S. Bhargava, K. R. Vasuki, and T. G. Sreeramamurthy, "Some evaluations of Ramanujan's cubic continued fraction," Indian Journal of Pure and Applied Mathematics, vol. 35, no. 8, pp. $1003-$ 1025, 2004.

[8] H. H. Chan, “On Ramanujan's cubic continued fraction," Acta Arithmetica, vol. 73, no. 4, pp. 343-355, 1995.

[9] K. R. Vasuki, A. A. Abdulrawf Kahatan, and G. Sharath, "On the series expansion of the Ramanujan cubic continued fraction," International Journal of Mathematical Combinatorics, vol. 4, pp. 84-95, 2011.

[10] K. R. Vasuki, N. Bhaskar, and G. Sharath, "On a continued fraction of order six," Annali dell'Universitá di Ferrara, vol. 56, no. 1, pp. 77-89, 2010.

[11] C. Adiga, B. C. Berndt, S. Bhargava, and G. N. Watson, "Chapter 16 of Ramanujan's second notebook: theta functions and $q$ series," Memoirs of the American Mathematical Society, vol. 315, pp. 1-91, 1985.

[12] C. Adiga and N. S. A. Bulkhali, "Identities for certain products of theta functions with applications to modular relations," Journal of Analysis \& Number Theory, vol. 2, no. 1, pp. 1-15, 2014.

[13] C. F. Gauss, "Hundert Theorems über die neuen Transscendenten," in Werke, 3, pp. 461-469, Königliche Gesellschaft der Wissenschaften zu Göttingen, Göttingen, Germany, 1876.

[14] Z.-G. Liu, "A three-term theta function identity and its applications," Advances in Mathematics, vol. 195, no. 1, pp. 1-23, 2005.

[15] S. McCullough and L.-C. Shen, "On the Szego" kernel of an annulus," Proceedings of the American Mathematical Society, vol. 121, no. 4, pp. 1111-1121, 1994.

[16] Z.-G. Liu, "A theta function identity and its implications," Transactions of the American Mathematical Society, vol. 357, no. 2, pp. 825-835, 2005. 


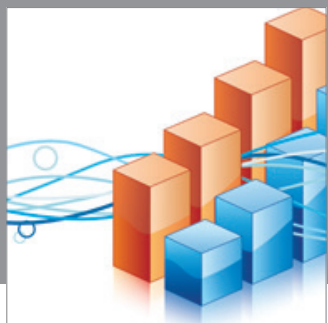

Advances in

Operations Research

mansans

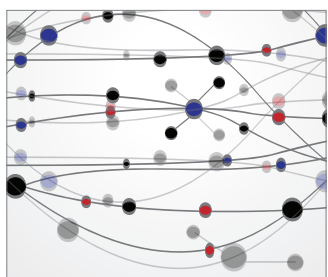

The Scientific World Journal
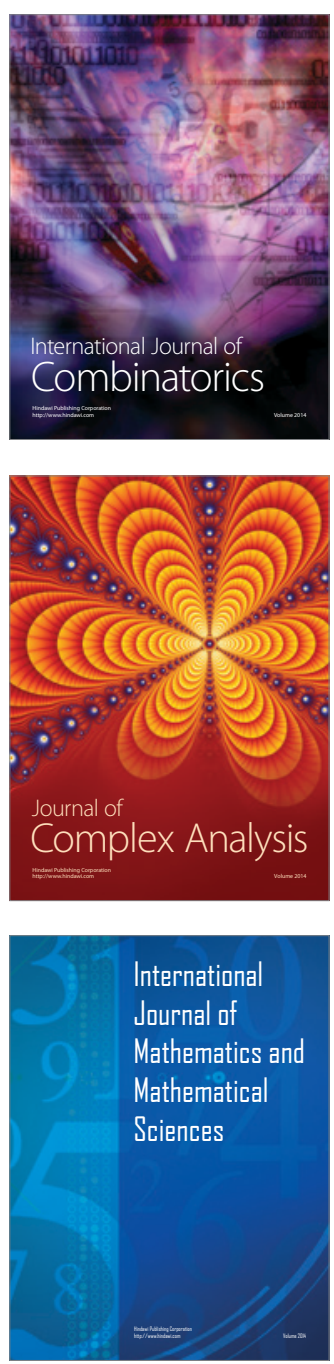
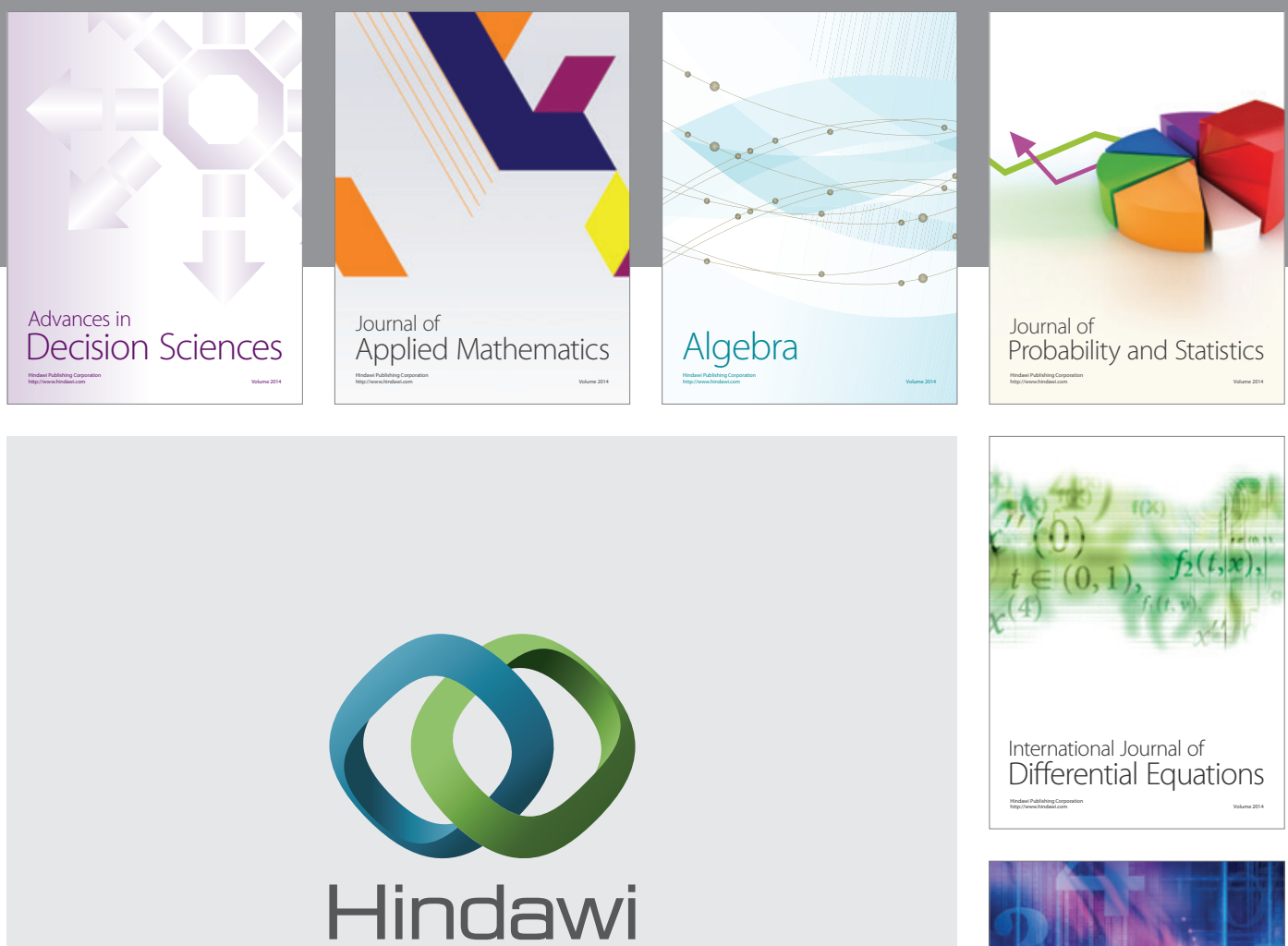

Submit your manuscripts at http://www.hindawi.com
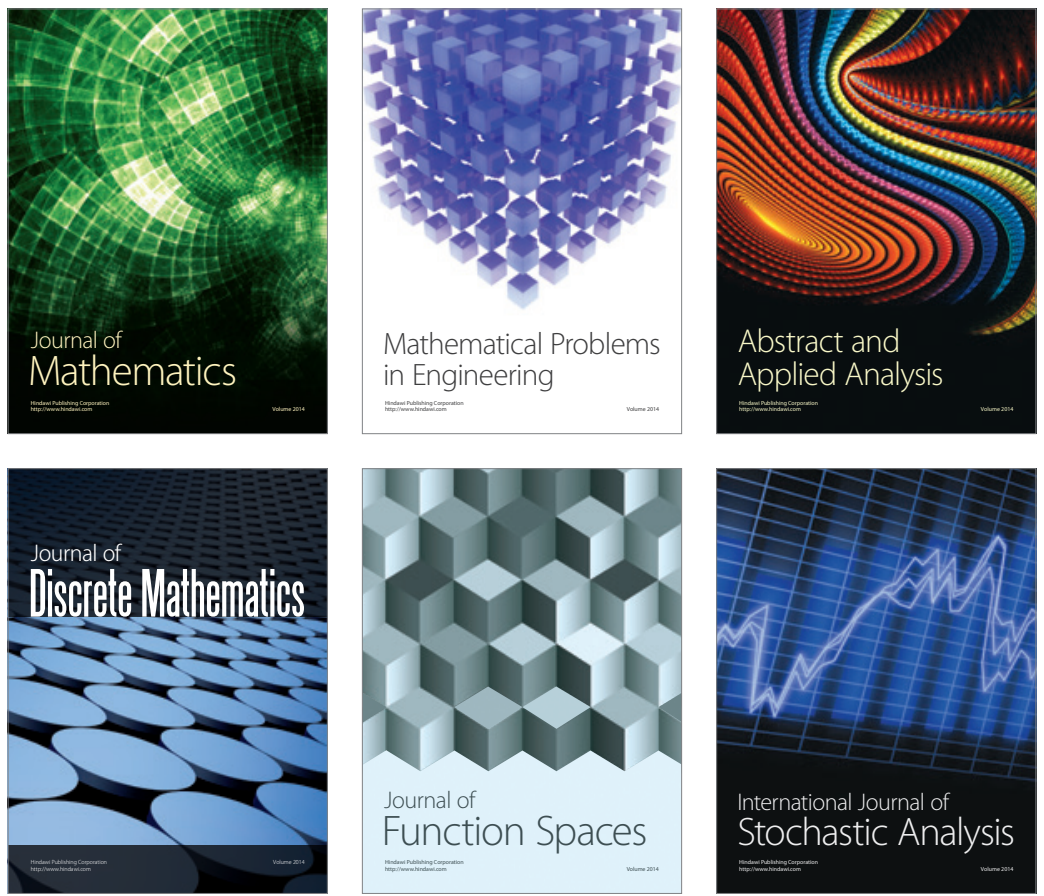

Journal of

Function Spaces

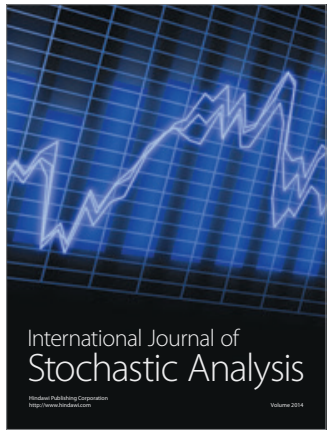

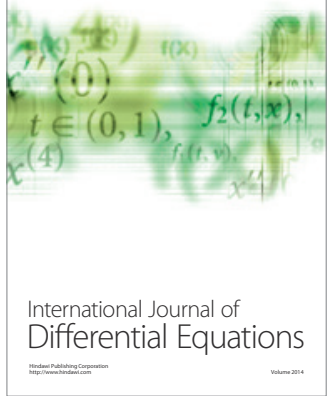
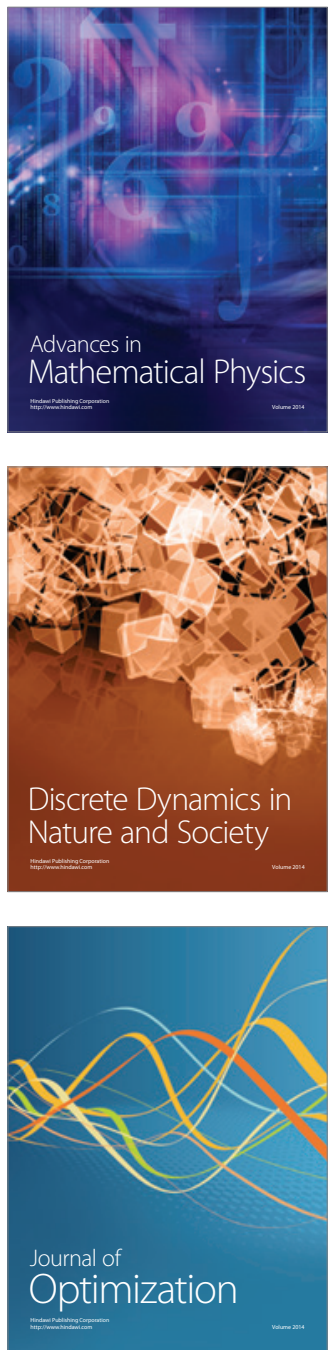